Document downloaded from:

http://hdl.handle.net/10251/50775

This paper must be cited as:

Reig, J.; Rubio Arjona, L. (2013). Estimation of the composite fast fading and shadowing distribution using the log-moments in wireless communications. IEEE Transactions on Wireless Communications. 12(8):3672-3681. doi:10.1109/TWC.2013.050713.120054.

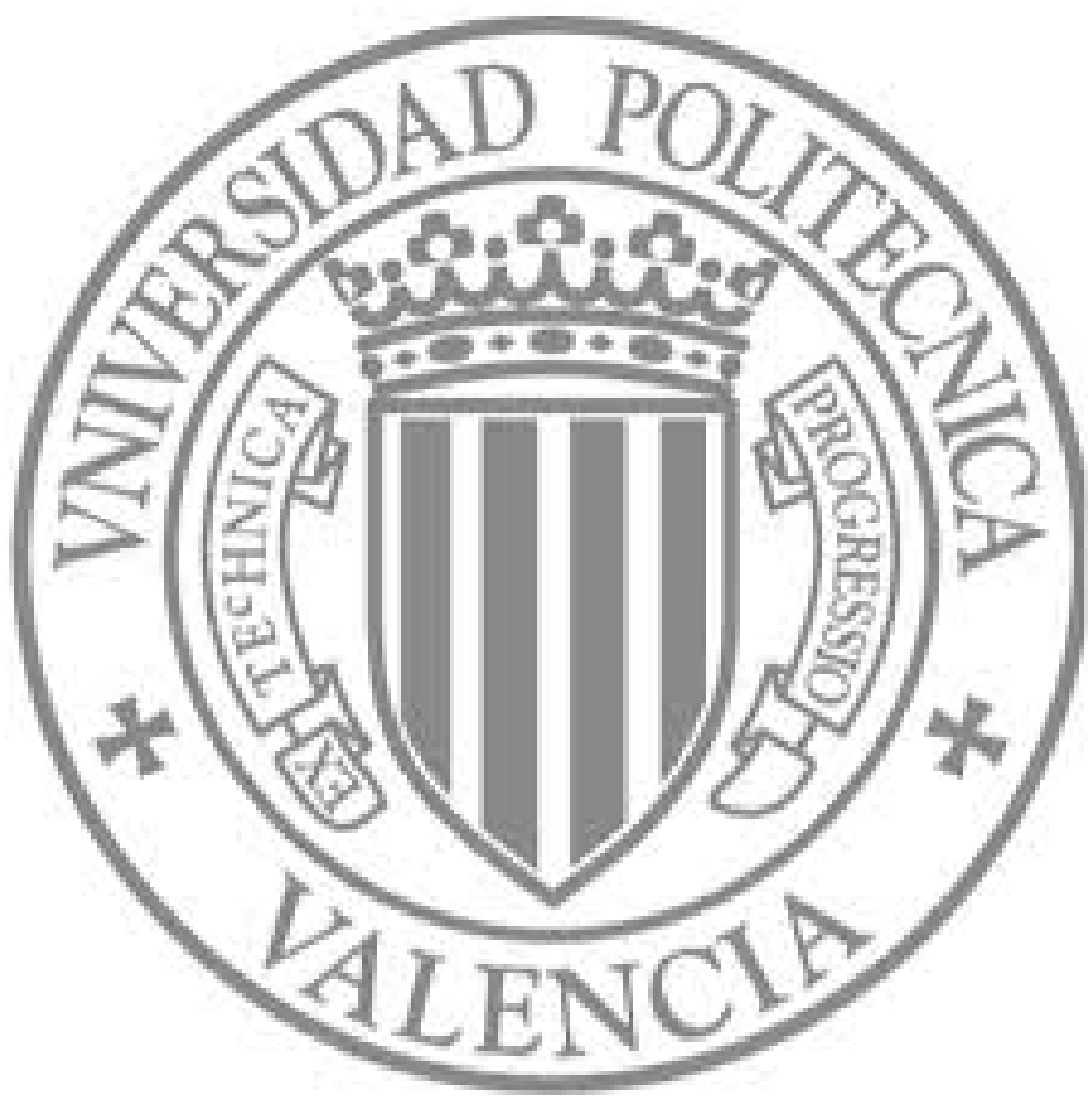

The final publication is available at

http://dx.doi.org/10.1109/TWC.2013.050713.120054

Copyright Institute of Electrical and Electronics Engineers (IEEE) 


\title{
Estimation of the Composite Fast Fading and Shadowing Distribution Using the Log-moments in Wireless Communications
}

\author{
Juan Reig, Member, IEEE, and Lorenzo Rubio, Member, IEEE
}

\begin{abstract}
In this work, we propose a framework to obtain estimators from a variety of distributions used in composite fast fading and shadowing modeling with applications in wireless communications: the Suzuki (Rayleigh-lognormal), Nakagamilognormal, $K$ (Rayleigh-gamma), generalized- $K$ (Nakagamigamma) and $\alpha-\mu$ (generalized gamma) distributions. These estimators are derived from the method of moments of these distributions in logarithmic units, usually known as log-moments. The goodness-of-fit of these estimators to experimental distributions has been checked from a measurement campaign carried out in an urban environment. Moreover a new method to separate fast fading and shadowing based on the Rathgeber procedure is proposed. The results conclude that the best-fitting distribution to the measurements is the Nakagami-lognormal. Also, the $\alpha-\mu$ distribution provides an acceptable matching with the advantage of its simplicity.
\end{abstract}

Index Terms-Mobile radio, fading, shadowing, estimator

\section{INTRODUCTION}

In wireless communications, the characterization of the received signal variability is a fundamental topic which has attracted the attention of numerous researchers [1], [2]. The received field strength experiences fading due to two factors. On the one hand, the received signal fluctuates deeply in a small local area around the receiver (typically in few wavelengths covered) which is the well-known short-term fading or small-scale fading phenomenon caused by the multipath propagation. In the literature, this small-scale fading has been characterized using the Rayleigh, Rice, Weibull and Nakagami- $m$ distributions [1], [3]. The Nakagami- $m$ statistical model has been extensively used in the literature, since the Nakagami- $m$ distribution matches the experimental data [4][6] and it includes the Rayleigh distribution as a specific case. On the other hand, for displacements of hundred of wavelengths, the received signal suffers slow variations due to the propagation shadowing of the direct path between the transmitter and receiver. These slow variations are the wellknown long-term fading or shadowing which have been mainly modeled using the lognormal distribution [7], [8].

The separation of the two fading contributions, i.e., the short and long-term fading, is rather troublesome, specially if the local mean of the received signal rapidly oscillates such as in indoor environments or in a situation of turning around

Manuscript received January 10, 2012; revised July 20, 2012, and December, 4; and accepted February 27, 2013.

J. Reig and L. Rubio are with the Electromagnetic Radiation Group (GRE) at the Universitat Politècnica de València, Camino de Vera s/n, 46022 Valencia, Spain. Email: jreigp@dcom.upv.es a corner in outdoor scenarios. Recently, an important effort have been carried out to characterize scenarios as vehicularto-vehicular (V2V) communications, where the assumption of stationary is not fulfilled [9]-[11].

Thus, the fast fading and shadowing compound distribution has been thoroughly analyzed in the technical literature [2], [12]-[21]. The most employed composite distribution is the Rayleigh-lognormal or Suzuki distribution [2], [12]. In [22][24] the estimation of the two parameters of the Suzuki distribution was derived using the method of log-moments. Moreover the Nakagami-lognormal distribution has been frequently employed for analyzing the fade depth and the performance parameters using different modulation schemes in wireless fading channels [13]-[16]. Nevertheless other distributions have been recently proposed to model the composite fast fading and shadowing [17]-[21], [25], since the Suzuki and the Nakagami-lognormal probability density functions (PDFs) cannot be expressed in simple forms and they are usually evaluated using integral formulae. The $K$ and the generalized$K$ distributions have been proposed to model the compound small-scale fading and shadowing [17]-[20]. The use of the gamma distribution to characterize the shadowing in such distribution provides the advantage that it leads to closedform expressions for the PDFs and cumulative distribution functions (CDFs). The generalized- $K$ distribution [17], [18] is equivalent to the Nakagami-gamma distribution and it is similar to the gamma-gamma or gamma- $K$ model described in the radar literature [26], [27]. The $K$ or the Rayleigh-gamma distribution used in [19], [20] to characterize the composite fading is a special case of the generalized- $K$ distribution.

The $\alpha-\mu$ distribution has been derived to characterize the fading in non-linear environments [28], [29]. This distribution is a form of the well-known generalized gamma or Stacy distribution [30] with considerable simpler PDF than Nakagamilognormal or Suzuki distributions. The $\alpha-\mu$ was employed in [25] to model the composite fast fading and shadowing distribution. Moreover in [21], the authors have mathematically derived a comprehensive collection of models obtained by compounding the Nakagami- $m$ with 16 distributions.

Several studies have been carried out to estimate the parameters of the composite fast fading and shadowing distribution in wireless communications from experimental data collected in measurement campaigns [1], [9], [25], [31]. In [1] the authors demonstrated that the Suzuki distribution fits better than both the Nakagami- $m$ and Weibull distributions in the 168, 455 and $900 \mathrm{MHz}$ frequency bands in London. 
In [25] the parameters of the $\alpha-\mu$, Suzuki, Nakagami- $m$, Rice and lognormal distributions were obtained and the statistical Kolmogorov-Smirnov (K-S) test was used to evaluate the goodness-of-fit of above distributions. The Weibull-lognormal estimators were derived in [31] to analyze the results of a measurement campaign carried out in Rio de Janeiro. The results of these measurements provide a better fitting of the Weibull-lognormal than both the Suzuki and Gaussian distributions. Recently, in [9] from a measurement campaign in a multiple-inputmultiple-output (MIMO) V2V scenario, the authors have modeled the long-term fading as lognormally distributed, whereas the small-scale fading is characterized by the Weibull distribution.

The method of moments applied over the distribution in logarithmic units is known as the log-moments or cumulants estimation method. The log-moments method has been proposed in synthetic aperture radar (SAR) image processing to estimate the amplitude distribution [32], [33]. In [23], [24] the comparison of the method of moments with the log-moments estimators has been analyzed and discussed particularized for the Suzuki distribution. The results of [23], [24] showed that estimates based on log-moments are generally superior to the method of moments. Other distributions have been proposed to characterize the composite fading effect such as the multiplicative $N$ Rayleigh and $N$ Nakagami- $m$ [34] distributions. However, Andersen showed in [35] that the $N$ Rayleigh distribution does not fit measurements carried out in a forest environment. That is why a multiple scattering model with physical motivation was proposed in [35] which provides a better fit to the measurements than the Nakagami- $m$ distribution in an indoor scenario [36]. Nevertheless, the derivation of estimators for the statistical distribution corresponding to this model is cumbersome following the log-moments approach.

Nevertheless, to the best of the authors' knowledge no estimators of Nakagami-lognormal, $K$ or generalized- $K$ distributions have been obtained from measurement campaigns. Moreover a derivation of Nakagami-lognormal distribution estimators remains open in the technical literature. Furthermore the separation of the fast fading and shadowing has not adequately been analyzed from a measured data yet [37]. To cover these gaps, in this work we propose an analytical framework to estimate the parameters of several statistical distributions based on the log-moments: the generalized- $K$, $K$, Nakagami-lognormal, Suzuki and $\alpha-\mu$. Moreover since the received signal measurements data are usually expressed in logarithmic units, e.g., $\mathrm{dBm}, \mathrm{dB} \mu \mathrm{V} / \mathrm{m}$, we derive the PDF and $\mathrm{CDF}$ of above analytical distributions in logarithmic units. Furthermore the goodness-of-fit of these distributions are evaluated in eight routes covered in an urban environment in the city of Valencia, in Spain, using the K-S test. A method based on the Rathgeber analysis [37] is also proposed to estimate an average window size for extracting the fast fading parameters from a measurement data record.

This paper is organized as follows: firstly in Section II we expose the relevant characteristics of the measurement campaign used in the derivation of the fading distribution estimators. Next the PDF, CDF and estimators of generalized$K, K$, Nakagami-lognormal, Suzuki and $\alpha-\mu$ distributions are derived in Section III. In Section IV we propose a fast fading extracting procedure based on the Rathgeber method. Numerical results are analyzed in Section V. Finally the conclusions are discussed in Section VI.

\section{Measurement Campaign}

A measurement campaign at $900 \mathrm{MHz}$ band was carried out in the urban area of Valencia, Spain. The received signal field strength was measured along eight routes with a total length of about $5 \mathrm{~km}$. The measurement setup was a narrowband power meter connected to a vertically polarized antenna mounted on a van and synchronized with a navigation system. The geographic information reported by the navigation system was used to identify the measured route on a digital map. Fig. 1 shows the digital map of the urban area of Valencia, the location of the two transmitters used in this campaign and the routes where the measurements were performed depicted by thick lines. The received signals along routes \#3, \#6, \#7 and \#8 arrived from the transmitter \#1 whereas the signals along routes \#1, \#2, \#4 and \#5 came from transmitter \#2.

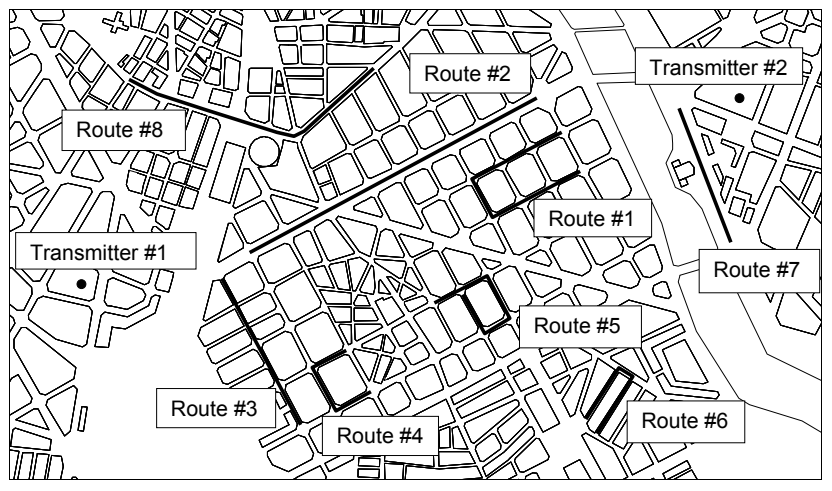

Fig. 1. Map of Valencia urban area. Thick lines depict the routes measured.

The heights of the transmitting antennas were $35 \mathrm{~m}$ from the ground. The measurements were carried out at nights to facilitate a constant speed of the van of about $20 \mathrm{~km} / \mathrm{h}$. With this speed, the spatial resolution of the measurement was about $\lambda / 4$ (distance between two samples).

\section{Estimators OF LOG Distributions}

\section{A. Generalized-K Distribution}

The PDF of the Nakagami- $m$ fading distribution conditioned on a shadowing distribution is given by

$$
\begin{array}{r}
p_{r \mid \Omega}(r \mid \Omega)=\frac{2}{\Gamma(m)}\left(\frac{m}{\Omega}\right)^{m} r^{2 m-1} \exp \left(-\frac{m r^{2}}{\Omega}\right), \\
r \geq 0, \Omega \geq 0,
\end{array}
$$

where $m$ is the fading parameter, $\Omega$ is the local power which models the shadowing variation, and $\Gamma(x)=$ $\int_{0}^{\infty} t^{x-1} \exp (-t) d t$ is the gamma function [38, (6.1.1)].

We assume that this short-term power, $\Omega$, experiences a gamma variation due to the shadowing, whose PDF is written as

$$
p_{\Omega}(\Omega)=\frac{1}{\Gamma(M)}\left(\frac{1}{\Omega_{0}}\right)^{M} \Omega^{M-1} \exp \left(-\frac{\Omega}{\Omega_{0}}\right) \quad \Omega \geq 0,
$$


where $M$ is the order of the gamma distribution and $E[\Omega]=$ $M \cdot \Omega_{0}$, with $E[\cdot]$ denoting expectation.

The PDF of the composed small-scale fading and shadowing distribution can be expressed as

$$
p_{r}(r)=\int_{0}^{\infty} p_{r \mid \Omega}(r \mid \Omega) p_{\Omega}(\Omega) d \Omega \quad r \geq 0 .
$$

Substituting (1) and (2) into (3) the generalized- $K$ or gamma-gamma distribution PDF is easily calculated as [39]

$$
p_{r}(r)=\frac{2 b}{\Gamma(m) \Gamma(M)}\left(\frac{b r}{2}\right)^{M+m-1} K_{M-m}(b r), r \geq 0,
$$

where

$$
b=2 \sqrt{\frac{m}{\Omega_{0}}},
$$

with $K_{x}(\cdot)$ being the modified Bessel function of the second kind [40, (8.407)].

The $k$-moment of this distribution is given by [18, (7)]

$$
E\left(r^{k}\right)=\frac{\Gamma\left(m+\frac{k}{2}\right) \Gamma\left(M+\frac{k}{2}\right)}{\Gamma(m) \Gamma(M)}\left(\frac{2}{b}\right)^{k} .
$$

The CDF of (4) can be calculated as [41]

$$
\begin{aligned}
& F_{r}(r)=\pi \csc (\pi(m-M))\left(\frac{1}{\Gamma(m)}\left(\frac{m r^{2}}{\Omega_{0}}\right)^{M}\right. \\
& { }_{1} F_{2}\left(M ; M+1, M-m+1, \frac{m r^{2}}{\Omega_{0}}\right)-\frac{1}{\Gamma(M)}\left(\frac{m r^{2}}{\Omega_{0}}\right)^{m} \\
& \left.{ }_{1} F_{2}\left(m ; m+1, m-M+1, \frac{m r^{2}}{\Omega_{0}}\right)\right), r \geq 0,
\end{aligned}
$$

where ${ }_{p} F_{q}(\cdot)$ is the generalized hypergeometric function [40, (9.14 1)], with $p, q$ positive integers; and csc symbolizes the cosecant function. Using the transformation $\epsilon=A \ln r$, where

$$
A=\frac{20}{\ln 10},
$$

the PDF of the log generalized- $K$ distribution can be obtained as

$$
\begin{aligned}
p_{\epsilon}(\epsilon)= & \frac{4}{A \Gamma(m) \Gamma(M)}\left(\frac{b \exp \left(\frac{\epsilon}{A}\right)}{2}\right)^{M+m} \\
& K_{M-m}\left(b \exp \left(\frac{\epsilon}{A}\right)\right),-\infty<\epsilon<\infty .
\end{aligned}
$$

From (9) and using [40, (6.561 16)] the moment-generating function (MGF) of the log generalized- $K$ distribution is derived as

$$
\mathcal{M}_{\epsilon}(s)=\frac{\Gamma\left(m+\frac{s}{2}\right) \Gamma\left(M+\frac{s}{2}\right)}{\Gamma(m) \Gamma(M)}\left(\frac{\Omega_{0}}{m}\right)^{\frac{s}{2}} .
$$

The $k$-moment of the log $K$-gamma distribution can be obtained by differentiating $k$ times the MGF given by (10) for $s=0$, following [42, p. 62] given by

$$
E\left[\epsilon^{k}\right]=\left.\frac{\partial^{k} \mathcal{M}_{\epsilon}(s)}{\partial s^{k}}\right|_{s=0} .
$$

Using (10) and (11) the second and third central moments of the $\log K$-gamma distribution can be calculated as

$$
\begin{aligned}
& \mu_{2}=E\left[(\epsilon-\bar{\epsilon})^{2}\right]=\left(\frac{A}{2}\right)^{2}\left(\psi^{\prime}(m)+\psi^{\prime}(M)\right), \\
& \mu_{3}=E\left[(\epsilon-\bar{\epsilon})^{3}\right]=\left(\frac{A}{2}\right)^{3}\left(\psi^{\prime \prime}(m)+\psi^{\prime \prime}(M)\right),
\end{aligned}
$$

where $\psi^{\prime}(x)=\frac{\partial \psi(x)}{\partial x}=\frac{\partial^{2} \ln \Gamma(x)}{\partial x^{2}}$ and $\psi^{\prime \prime}(x)=\frac{\partial^{2} \psi(x)}{\partial x^{2}}=$ $\frac{\partial^{3} \ln \Gamma(x)}{\partial x^{3}}$ are the polygamma functions of first and second order, respectively $[38,(6.4 .1)] \psi(x)=\frac{\partial \ln \Gamma(x)}{\partial x}$ is the psi (digamma) function $[38,(6.3 .1)]$; and $A$ is given by (8). We can use equations (12)-(13) to estimate $\hat{m}$ and $\hat{M}$ parameters using numerical methods. Once $M$ is calculated, we can estimate $\hat{\Omega}_{0}$ using

$$
\Omega_{0}=\frac{E\left[r^{2}\right]}{M},
$$

where (14) has been calculated by substituting $k=2$ in (6), with $b$ defined in (5). The $K$ distribution corresponds to a Rayleigh-gamma model. Therefore, we can substitute $m=1$ in (4) to obtain the PDF of the $K$ distribution. Thus, substituting $m=1$ in (12) and using [40, (8.366 8)] $\psi^{\prime}(1)=\pi^{2} / 6$, the second central moment is obtained as

$$
\mu_{2}=E\left[(\epsilon-\bar{\epsilon})^{2}\right]=\left(\frac{A}{2}\right)^{2}\left(\psi^{\prime}(M)+\frac{\pi^{2}}{6}\right) .
$$

We can use the Lorenz estimator [22] obtained for the Nakagami- $m$ distribution to calculate $\hat{M}$ from (15). This Lorenz estimator makes use of an approximation of the inverse polygamma function of the first order as

$$
u=\left(\frac{A}{2}\right)^{2} \psi^{\prime}(x) \longrightarrow x=u^{-1} \approx \frac{4.4}{\sqrt{u}}+\frac{17.4}{u^{1.29}},
$$

where $u$ is a function of $x$ and $u^{-1}$ symbolizes the inverse of the $u$ function. Combining (15) and (16) we can estimate $\hat{M}$ as

$$
\hat{M}=\frac{4.4}{\sqrt{\hat{\mu}_{2}-B}}+\frac{17.4}{\left(\hat{\mu}_{2}-B\right)^{1.29}},
$$

where $\hat{\mu}_{2}$ is an estimator of the second central moment of the $\log K$ distribution, and

$$
B=\left(\frac{A}{2}\right)^{2} \frac{\pi^{2}}{6}=31.02,
$$

is the variance of the log Rayleigh distribution. Therefore, $\hat{M}$ is consistent for $\hat{\mu}_{2}>31.02$. In other words, the Rayleighgamma distribution in logarithmic units always has a variance higher than 31.02 or equivalently a standard deviation larger than $5.57 \mathrm{~dB}$.

\section{B. Nakagami-lognormal Distribution}

Let $r \mid \Omega$ be a conditional random variable (RV) Nakagami- $m$ distributed whose PDF is given by (1). If the local power which models the shadowing variation, $\Omega$, is assumed lognormal distributed then its PDF is given by

$$
p_{\Omega}(\Omega)=\frac{1}{\sqrt{2 \pi} \sigma \Omega} \exp \left(-\frac{(\ln \Omega-\eta)^{2}}{2 \sigma^{2}}\right), \quad \Omega \geq 0,
$$


where $\frac{10}{\ln 10} \eta$ and $\frac{10}{\ln 10} \sigma$ are the mean in $\mathrm{dBV} / \mathrm{m}$ and the standard deviation in $\mathrm{dB}$ of the associated Gaussian process, respectively, corresponding to the lognormal shadowing. The PDF of the Nakagami-lognormal distribution can be obtained by substituting (1) and (19) into (3) as [14]

$$
\begin{aligned}
& p_{r}(r)=\int_{0}^{\infty} \frac{2}{\Gamma(m)}\left(\frac{m}{\Omega}\right)^{m} r^{2 m-1} \exp \left(-\frac{m r^{2}}{\Omega}\right) \\
& \frac{1}{\sqrt{2 \pi} \sigma \Omega} \exp \left(-\frac{(\ln \Omega-\eta)^{2}}{2 \sigma^{2}}\right) d \Omega,
\end{aligned}
$$

where $m$ is the fading parameter of the Nakagami- $m$ fading process.

The CDF of the Nakagami-lognormal distribution can be calculated using $[40,(3.3262)]$ as

$$
\begin{aligned}
& F_{r}(r)=\int_{0}^{\infty} \frac{1}{\Gamma(m)} \gamma\left(m, \frac{m}{\Omega} r^{2}\right) \\
& \frac{1}{\sqrt{2 \pi} \sigma \Omega} \exp \left(-\frac{(\ln \Omega-\eta)^{2}}{2 \sigma^{2}}\right) d \Omega
\end{aligned}
$$

where $\gamma(a, x)=\int_{0}^{x} t^{a-1} \exp (-t) d t$ is the lower incomplete gamma function $[38,(6.5 .2)]$. We can express the PDF of the log Nakagami-lognormal distribution as

$$
\begin{aligned}
& p_{\epsilon}(\epsilon)=\int_{0}^{\infty} \frac{2}{A \Gamma(m)}\left(\frac{m}{\Omega}\right)^{m} \exp \left(\frac{2 m \epsilon}{A}-\frac{m e^{\frac{2 \epsilon}{A}}}{\Omega}\right) \\
& \frac{1}{\sqrt{2 \pi} \sigma \Omega} \exp \left(-\frac{(\ln \Omega-\eta)^{2}}{2 \sigma^{2}}\right) d \Omega,
\end{aligned}
$$

with $\epsilon=A \ln r$ and $A$ given by (8). The MGF of the $\log$ Nakagami-lognormal distribution can be obtained as [23, (3.6)]

$$
\mathcal{M}_{\epsilon}(s)=\frac{\Gamma\left(m+\frac{A s}{2}\right)}{m^{\frac{A s}{2}} \Gamma(m)} \exp \left(\frac{1}{8} A s\left(4 \eta+A \sigma^{2} s\right)\right) .
$$

The first moment is easily obtained using (11) and (23) as

$$
m_{1}=E[\epsilon]=\frac{A}{2}(\eta-\ln m+\psi(m)),
$$

and the second and third central moments are also derived as

$$
\begin{aligned}
& \mu_{2}=E\left[(\epsilon-\bar{\epsilon})^{2}\right]=\left(\frac{A}{2}\right)^{2}\left(\sigma^{2}+\psi^{\prime}(m)\right), \\
& \mu_{3}=E\left[(\epsilon-\bar{\epsilon})^{3}\right]=\left(\frac{A}{2}\right)^{3} \psi "(m) .
\end{aligned}
$$

From (26) if we define $u=\psi "(m)=(2 / A)^{2} \mu_{3}$, we can calculate an approximation of $m=u^{-1}$ using numerical methods. Fig. 2 shows in solid line the numerical $u$ function for a relative error less than $10^{-4}$ with $u$ from -16.83 to -0.001 . The minimum value of $u=-16.83$ corresponds to $m=0.5$, which is the minimum possible value of the Nakagami- $m$ fading parameter. Note that both axes are in logarithmic units. The behavior of this function suggests that $\ln m$ can be approximated in this range of values with a polynomial as a function of $\ln u$. Consequently the approximate polynomial has been derived using the least-squares methods as

$$
y=0.0096 x^{2}-0.391 x+0.3365,
$$

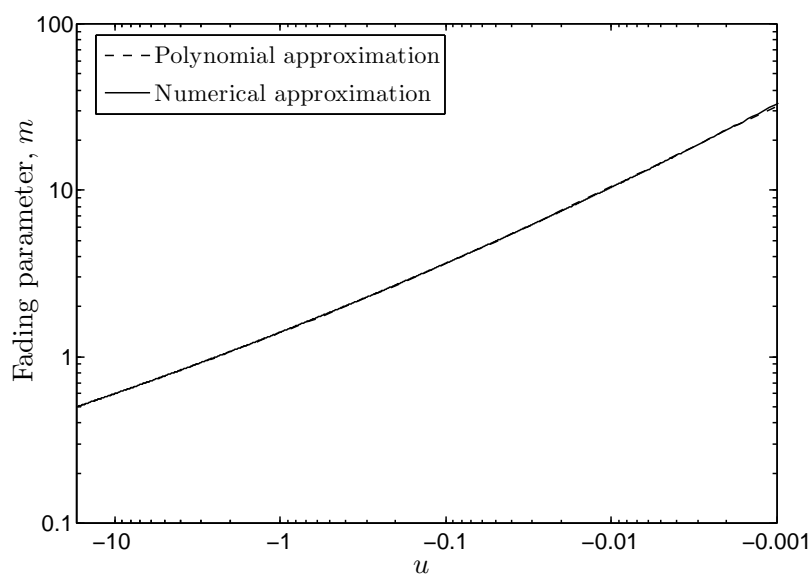

Fig. 2. Fading parameter, $m$, of the Nakagami-lognormal distribution as a function of the $u$ function.

where $y=\ln m$ and $x=\ln u$. In Fig. 2 this polynomial is plotted with dashed lines and it shows an almost perfect fit. Thus, using (27) we can calculate an approximation of $\hat{m}$ as

$$
\hat{m}=1.4|\hat{u}|^{-0.391+0.0096 \ln |\hat{u}|},-16.8 \leq \hat{u}<0,
$$

where

$$
\hat{u}=\left(\frac{2}{A}\right)^{3} \hat{\mu}_{3},
$$

with $\hat{\mu}_{3}$ being an estimator of the third central moment of the log Nakagami-lognormal distribution, and $|\cdot|$ the modulus operator. The rest of parameters of the Nakagami-lognormal distribution, $\hat{\eta}$ and $\hat{\sigma}$, can be estimated from (24) and (25), respectively. In Fig. 3 the relative error expressed in \% of the

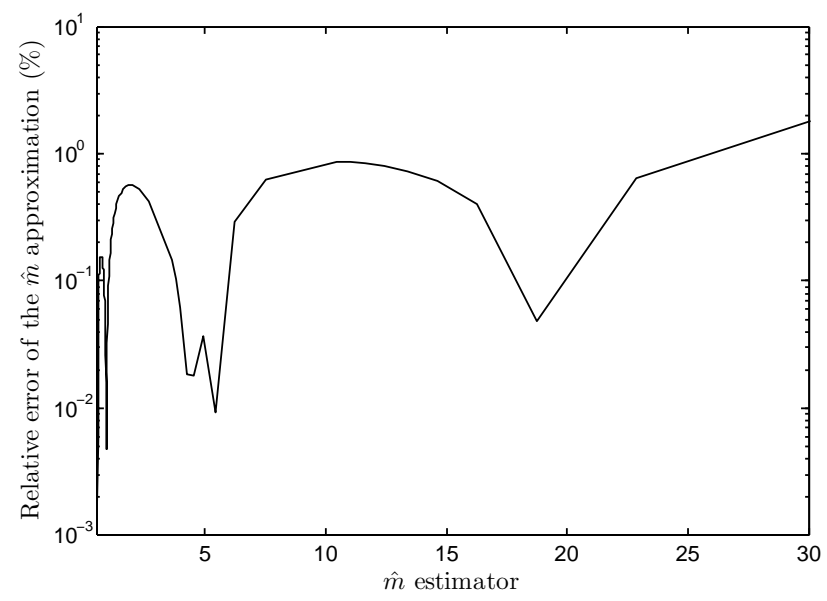

Fig. 3. Relative error of the $\hat{m}$ approximation as a function of $\hat{m}$ for the Nakagami-lognormal distribution.

$\hat{m}$ approximation given by (28) is plotted in terms of the $\hat{m}$ estimator. The relative error of the $\hat{m}$ approximation is less than $0.56 \%$ for $\hat{m}$ varying from 0.5 to 7 . Fading parameters found in real environments [43] are inside this interval. For the Suzuki or Rayleigh-lognormal distribution [2], [12], we can estimate $\hat{\eta}$ and $\hat{\sigma}$, by substituting $m=1$ in (24) and (25) 
as [23]-[25]

$$
\begin{aligned}
& \hat{\eta}=\frac{2}{A} \hat{m}_{1}+C, \\
& \hat{\sigma}=\sqrt{\left(\frac{2}{A}\right)^{3} \hat{\mu}_{2}-\frac{\pi^{2}}{6},}
\end{aligned}
$$

where $C=0.577 \ldots$ is the Euler's constant [40, (9.73)]; and $\hat{m}_{1}$ and $\hat{\mu}_{2}$ being the estimators of the first moment and second central moment of the log Suzuki distribution, respectively.

\section{Generalized Gamma or $\alpha-\mu$ Distribution}

The PDF of the generalized gamma or $\alpha-\mu$ distribution is given by [30, (1)], [28]

$$
p_{r}(r)=\frac{\alpha \mu^{\mu} r^{\alpha \mu-1}}{\Omega^{\alpha \mu} \Gamma(\mu)} \exp \left(-\mu \frac{r^{\alpha}}{\Omega^{\alpha}}\right), r \geq 0, \alpha>0, \mu>0,
$$

where

$$
\begin{gathered}
\mu=\frac{E^{2}\left[r^{\alpha}\right]}{E\left[r^{2 \alpha}\right]-E^{2}\left[r^{\alpha}\right]}, \\
\Omega=E^{1 / \alpha}\left[r^{\alpha}\right] .
\end{gathered}
$$

The CDF of the $\alpha-\mu$ distribution is calculated as [28, (9)]

$$
F_{r}(r)=\frac{\Gamma\left(\mu, \mu \frac{r^{\alpha}}{\Omega^{\alpha}}\right)}{\Gamma(\mu)},
$$

where $\Gamma(a, x)=\int_{x}^{\infty} t^{a-1} \exp (-t) d t$ is the upper incomplete gamma function [38, (6.5.3)]. The PDF of the $\log \alpha-\mu$ distribution is calculated by applying the transformation $\epsilon=A \ln r$, with $A$ given by (8), in (32) as

$$
\begin{array}{r}
p_{\epsilon}(\epsilon)=\frac{1}{A} \frac{\alpha \mu^{\mu}}{\Omega^{\alpha \mu} \Gamma(\mu)} \exp \left(\frac{\alpha \mu}{A} \epsilon-\frac{\mu}{\Omega^{\alpha}} e^{\frac{\alpha \epsilon}{A}}\right), \\
-\infty<\epsilon<\infty .
\end{array}
$$

From (36) and using [40, (3.3814)], the MGF of the $\log \alpha-\mu$ distribution can be expressed as

$$
\mathcal{M}_{\epsilon}(s)=\left(\frac{\Omega^{\alpha}}{\mu}\right)^{\frac{A s}{\alpha}} \frac{\Gamma\left(\mu+\frac{A s}{\alpha}\right)}{\Gamma(\mu)} .
$$

Substituting (37) in (11) the second and third central moments of $\epsilon$ can be calculated from as

$$
\begin{aligned}
& \mu_{2}=E\left[(\epsilon-\bar{\epsilon})^{2}\right]=\left(\frac{A}{\alpha}\right)^{2} \psi^{\prime}(\mu), \\
& \mu_{3}=E\left[(\epsilon-\bar{\epsilon})^{3}\right]=\left(\frac{A}{\alpha}\right)^{3} \psi^{\prime \prime}(\mu) .
\end{aligned}
$$

We can define an estimator, $\hat{\kappa}$, according to [44] as

$$
\hat{\kappa}=\frac{\hat{\mu}_{2}^{3 / 2}}{\hat{\mu}_{3}} \triangleq \frac{\left(\psi^{\prime}(\mu)\right)^{3 / 2}}{\psi^{\prime \prime}(\mu)},
$$

where $\hat{\mu}_{2}$ and $\hat{\mu}_{3}$ are the estimators of the central moments $\mu_{2}$ and $\mu_{3}$ of the $\log \alpha-\mu$ distribution, respectively. From (40), the $\alpha$ parameter of the $\alpha-\mu$ distribution can be estimated as [45]

$$
\hat{\mu}=\left\{\begin{array}{lr}
\hat{\kappa}^{2}+\frac{1}{2} & \hat{\kappa} \leq-2.85 \\
-0.0773 \hat{\kappa}^{4}-0.6046 \hat{\kappa}^{3}-0.7949 \hat{\kappa}^{2} & \\
-2.4675 \hat{\kappa}-0.9208 & -2.85<\hat{\kappa} \leq-0.6 \\
-132.8995 \hat{\kappa}^{3}-232.0659 \hat{\kappa}^{2} & \\
-137.6303 \hat{\kappa}-27.3616 & -0.6<\hat{\kappa}<-0.5
\end{array}\right.
$$

From (34) and (38), an estimation of $\alpha$ and $\Omega$ parameters can be easily obtained as

$$
\begin{aligned}
& \hat{\alpha}=A \sqrt{\frac{\psi^{\prime}(\hat{\mu})}{\hat{\mu}_{2}}}, \\
& \hat{\Omega}=E^{1 / \hat{\alpha}}\left[\hat{r}^{\hat{\alpha}}\right] .
\end{aligned}
$$

The normalized mean square error of the estimators given by (41) and (42) is analyzed in [45].

\section{Separation of Fast Fading and Shadowing}

A statistical characterization of the received signal variations for both the small-scale fading and shadowing is useful to design the transmitter and receiver transceivers. Thus, the knowledge of small-scale fading statistics can be used to design specific micro-diversity techniques (e.g., spatial, temporal, polarization, etc.), coding and modulation schemes. On the one hand, the implementation of small-scale fading models permits us to evaluate the error probability in fast fading channels taking into account parameters as coherence time and Doppler spread that are related to small-scale variations of the signal. On the other hand, the knowledge of the large-scale variations is useful in power control techniques and in the evaluation of the coverage area. The separation of both types of fading permits us to develop and implement the proper technique to mitigate each type of fading in a separate way.

Since the small-scale fading and shadowing is characterized as a multiplicative effect $[1,(5.84)]$, the envelope of the received signal, $r$, can be written as

$$
r=r_{f} \cdot r_{s},
$$

where $r_{f}$ is the small-scale amplitude with a normalized power, $E\left[r_{f}^{2}\right]=1$; and $r_{s}$ is the shadowing envelope. Note that the PDF of the composite distribution of $\mathrm{r}$ can alternatively be expressed as (3). If we define $\epsilon=A \ln r, \epsilon_{f}=A \ln r_{f}$ and $\epsilon_{s}=A \ln r_{s}$ where $A$ is given by (8), the logarithmic composite amplitude, $\epsilon$, is calculated as [1, (5.85)]

$$
\epsilon=\epsilon_{f}+\epsilon_{s},
$$

Since both the small-scale fading and shadowing amplitudes are independent, the standard deviation of the compound fast fading and shadowing $\log$ distributions, denoted by $\sigma_{c}$ and expressed in $\mathrm{dB}$, is obtained as

$$
\sigma_{c}=\sqrt{\sigma_{f}^{2}+\sigma_{s}^{2}}
$$

where $\sigma_{f}$ and $\sigma_{s}$ are the standard deviations of the fast fading and shadowing amplitude distributions in $\mathrm{dB}$, respectively. 
TABLE I

STANDARD DEVIATION IN DB OF THE FAST-FAdING AND SHADOWING LOG DisTRIBUTIONS.

\begin{tabular}{ccccc}
\hline & Generalized- $\boldsymbol{K}$ & $\boldsymbol{K}$ & $\begin{array}{c}\text { Nakagami- } \\
\text { lognormal }\end{array}$ & Suzuki \\
\hline$\sigma_{f}$ & $\frac{10}{\ln 10} \sqrt{\psi^{\prime}(m)}$ & $\frac{10}{\ln 10} \frac{\pi}{\sqrt{6}}=5.57$ & $\frac{10}{\ln 10} \sqrt{\psi^{\prime}(m)}$ & $\frac{10}{\ln 10} \frac{\pi}{\sqrt{6}}=5.57$ \\
\hline$\sigma_{s}$ & $\frac{10}{\ln 10} \sqrt{\psi^{\prime}(M)}$ & $\frac{10}{\ln 10} \sqrt{\psi^{\prime}(M)}$ & $\sigma$ & $\sigma$ \\
\hline
\end{tabular}

The standard deviation of the logarithmic generalized gamma and derived distributions (Nakagami- $m$, Rayleigh and Weibull distributions) does not depend on the second raw moment of such distributions in linear units, i.e., $E\left[r_{f}^{2}\right]$. However the standard deviation of the logarithmic Rice distribution is related to the second raw moment of this distribution in linear units. Thus the variance of the logarithmic composite distribution is equivalent to the expressions (12) and (25) for the generalized- $K$ and Nakagami-lognormal distributions, respectively. In Table I, these standard deviations are shown as a function of the parameters of the distributions analyzed in Section III. Note that the standard deviation of the $\log \alpha$ $\mu$ or generalized gamma distribution cannot be separated in the short-term fading and shadowing terms. Nevertheless, the $\alpha$ and $\mu$ estimated parameters using the log-moments method are subject to limitations when the $\alpha-\mu$ distribution is used to model the composite small-scale fading and shadowing. It is of interest to analyze the range of the estimated $\alpha$ and $\mu$ values for wireless communications. Assuming that the fast fading is Nakagami- $m$ distributed, then $\sigma_{f}$ is given by $\frac{10}{\ln 10} \sqrt{\psi^{\prime}(m)}$ (see Table I) where $m$ is the fading parameter of the Nakagami- $m$ distribution. In measurements carried out in macrocellular environments, the fading parameter typically oscillates from 0.5 to 3.5 [43]. The standard deviation of the shadowing usually varies from 3 to $12 \mathrm{~dB}$ in macrocellular, microcellular and indoor environments [3]. Moreover, using the log-moments method the standard deviation of the composite distribution should be equal to the square root of the second central log-moment of the $\alpha-\mu$ distribution given by (38), i.e.,

$$
\hat{\sigma}_{c} \triangleq \frac{20}{\hat{\alpha} \ln 10} \sqrt{\psi^{\prime}(\hat{\mu})} \triangleq \sqrt{\left(\frac{10}{\ln 10}\right)^{2} \psi^{\prime}(\hat{m})+\hat{\sigma}_{s}^{2}} .
$$

From the third central log-moments of both $\alpha-\mu$ and Nakagami-lognormal distributions, we can find another limit for the $\alpha$ and $\mu$ parameters. From (26) and (39), we can easily obtain

$$
\hat{\mu}_{3} \triangleq\left(\frac{20}{\hat{\alpha} \ln 10}\right)^{3} \psi "(\hat{\mu}) \triangleq\left(\frac{10}{\ln 10}\right)^{3} \psi "(\hat{m}) .
$$

In Fig. 4 we have plotted a graph with the values of permitted $\alpha$ and $\mu$ for $m=0.5$ to 3.5 and $\sigma_{s}=0$ to $12 \mathrm{~dB}$ using (45) and (46). The region shaded in grey corresponds to the possible values of $\alpha$ and $\mu$ using the log-moments estimators which is limited for the four curves. Note that the $\alpha-\mu$ becomes a Weibull distribution for $\mu=1$ and the $\alpha-\mu$ converts into a Nakagami- $m$ distribution for $\alpha=2$ [28]. For both $\mu=1$ and $\alpha=2$ the $\alpha-\mu$ is equivalent to a Rayleigh distribution [28]. Recently, in [46] Shankar obtained boundaries for $\alpha$ values in

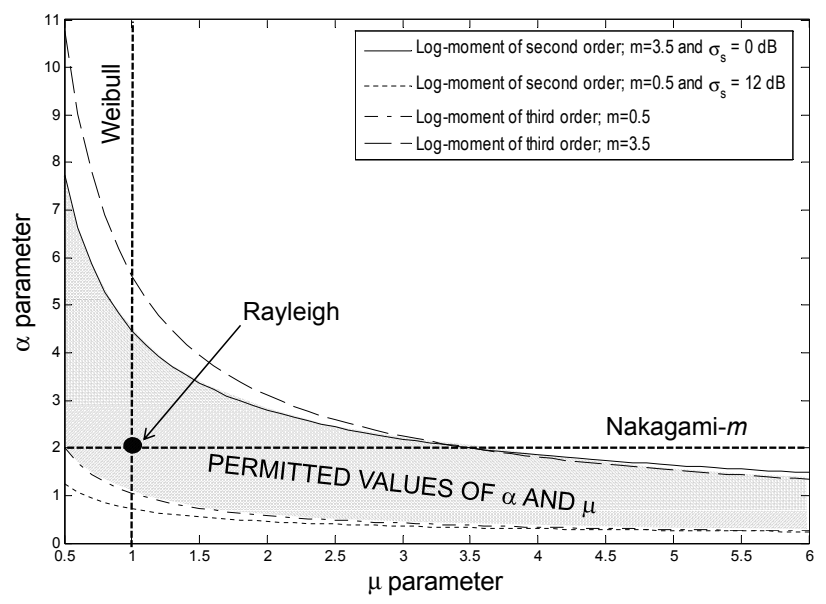

Fig. 4. Values of permitted $\alpha$ and $\mu$ parameters of the $\alpha-\mu$ distribution using the log-moments estimation method. Fading parameter for the smallscale distribution and shadowing standard deviation are $m=0.5$ to $3.5, \sigma_{s}=$ 0 to $12 \mathrm{~dB}$, respectively.

shadowed channels. This restriction assumes that the multipath physical model proposed by Yacoub in [29, (10)] is satisfied. This implies that $\mu$ is equal to the fading parameter, $m$, of the small-scale amplitude distribution. This condition applied to (47) leads to $0<\alpha<2$ [46, (65)]. Nevertheless from the results shown in Table III, the estimated fading parameters, $m$, of the Nakagami-lognormal distributions are substantially different from the $\mu$ estimated parameters in the measurements carried out in this paper. Thus the Yacoub's smallscale model $[29,(10)]$ cannot be applied to this environment and therefore Shankar's relationship given by [46, (65)], i.e., substituting $\mu=m$ in (47), is not fulfilled. Hence avoiding this restriction, $\alpha$ can be equal or greater than 2 due to the permitted values area shown in Fig. 4.

In [37], a method for the separation of the samples affected mainly by short-term fading with a local mean practically stable was suggested when there is a record data from measurements. This procedure proposes to calculate the standard deviation of a window extracted from a route expressed in logarithmic received field strength. In this method, the size of the window is increased until the standard deviation of the logarithmic samples is stabilized below and close to 5.57 $\mathrm{dB}$, which corresponds to the standard deviation of the $\log$ Rayleigh distribution, $\sigma_{f}=\frac{10}{\ln 10} \sqrt{\psi^{\prime}(1)}=\frac{10 \pi}{\ln 10 \sqrt{6}}=5.57$ $\mathrm{dB}$. This optimal size of the window assures that the received signal samples contained in this window are not substantially affected by shadowing. For window sizes larger than this optimal size, the received signal samples also suffer shadowing and the standard deviation increases until $\sigma_{c}$ given by (46). Nevertheless, the performance of this method depends strongly on the choice of the window center. If the samples around the windows center are subject to the shadowing effect the extraction of the short-term from the route is troublesome. In Fig. 5, two examples of the separation of the short-term fading and shadowing/ are illustrated for the route \#4. Fig. 5.a and Fig. 5.b show the standard deviation of the field strength in $\mathrm{dBV} / \mathrm{m}$ inside the window with $\lambda$ size. These samples are extracted from two windows illustrated in the Fig. 5.c, where 


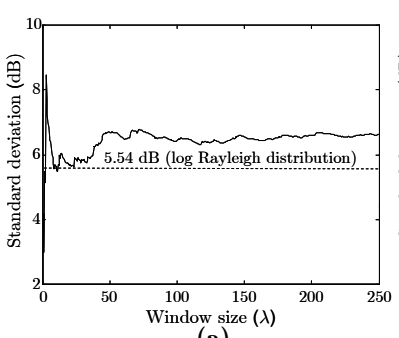

(a)

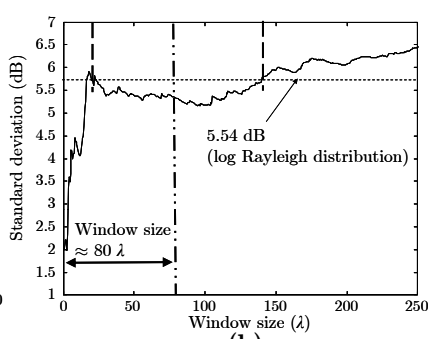

(b)

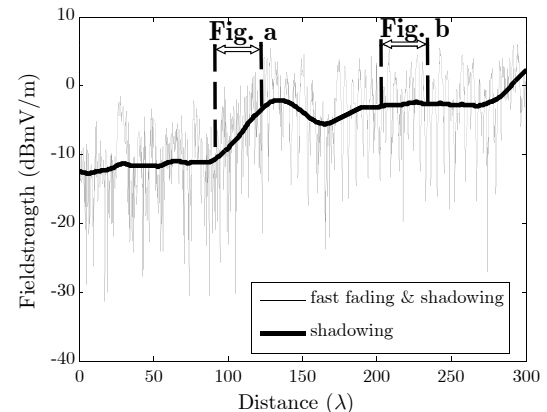

(c)

Fig. 5. Rathgeber method for separating both the short-term and long-term fading in two windows of the route \#4.

the field strength along the route \#4 is plotted as a function of the distance in $\lambda$. Fig. 5.b shows that this standard deviation is stabilized from approximately $20 \lambda$ up to $140 \lambda$ window sizes below and close to $5.57 \mathrm{~dB}$ (log Rayleigh standard deviation). Thus, the optimum window size can be estimated as the mean value from $20 \lambda$ to $140 \lambda$, yielding $80 \lambda$. Since in the center of the Fig. 5.b window the long-term field strength variation is negligible, the short-term fading and the shadowing can be easily separated using this method. Nevertheless in the example of Fig. 5.a, the extraction of the short-term fading is very difficult due to the shadowing varies substantially around the center of the window.

That is why we propose a new method for estimating an average window size along a route whose samples are not mainly subject to the shadowing variation. We assume that the small-scale and long-term processes are independent [47]. Once the window size is fixed, we can average the standard deviation of the field strength in $\mathrm{dBV} / \mathrm{m}$ along the route varying the center of the window for a given route. Thus, the averaged standard deviation in logarithmic units of the composite processes (short-term fading and shadowing) along a window of size $\Delta d$ can be easily calculated following a procedure similar to [47] as

$$
\bar{\sigma}_{\Delta d}=\sqrt{\sigma_{f}^{2}\left(1-C_{f}(\Delta d)\right)+\sigma_{s}^{2}\left(1-C_{s}(\Delta d)\right)}
$$

where $C_{f}(\cdot)$ and $C_{s}(\cdot)$ are the normalized auto-covariances of the small-scale and long-term fading in logarithmic units, respectively. Note that the shape of the averaged variance of a process along a window is similar to that of the structure function, used by Tatarski [48] and compiled in [49] to describe non-stationary random processes corresponding to wave propagation signals. This structure function is defined as $[49,(47)]$

$$
D_{\Delta d}=2\left(C_{p}(0)-C_{p}(\Delta d)\right)=2\left(1-C_{p}(\Delta d)\right),
$$

where $C_{p}(\cdot)$ is the normalized auto-covariance of the analyzed process. If the process is stationary this function tends to stabilize for high $\Delta d$ corresponding to low values of $C_{p}(\Delta d)$ (see Figs. 14 and 15 of [49]).

\section{NumericAl Results}

In Table III, using the log-moments derived in above equations the parameters of the $\alpha-\mu$, Nakagami-lognormal, Suzuki, generalized- $K$ and $K$ have been shown for the received field strength in all routes. Since the parameters of the generalized$K, m$ and $M$, in (12) and (13) are interchangeable, we have selected the minimum of both values to $m$ corresponding to the fading parameter of the short-term fading. Moreover, in the routes \#1, \#4, \#5 and \#8, the generalized- $K$ log-moments do not provide any solution. Thus, we have obtained the values of $\hat{m}$ and $\hat{M}$ which simultaneously minimize both $\hat{\mu}_{2}-$ $\left(\frac{A}{2}\right)^{2}\left(\psi^{\prime}(m)+\psi^{\prime}(M)\right)$ and $\hat{\mu}_{3}-\left(\frac{A}{2}\right)^{3}(\psi "(m)+\psi "(M))$. Note that for the $\alpha-\mu$ distribution, in Fig. 4 the estimated values of $\alpha$ and $\mu$ parameters of all routes are inside the shaded area in grey. For instance, the estimated values for the route \#1 are $\hat{\alpha}=0.45$ and $\mu=5.01$, which correspond to a dot inside the area of permitted values.

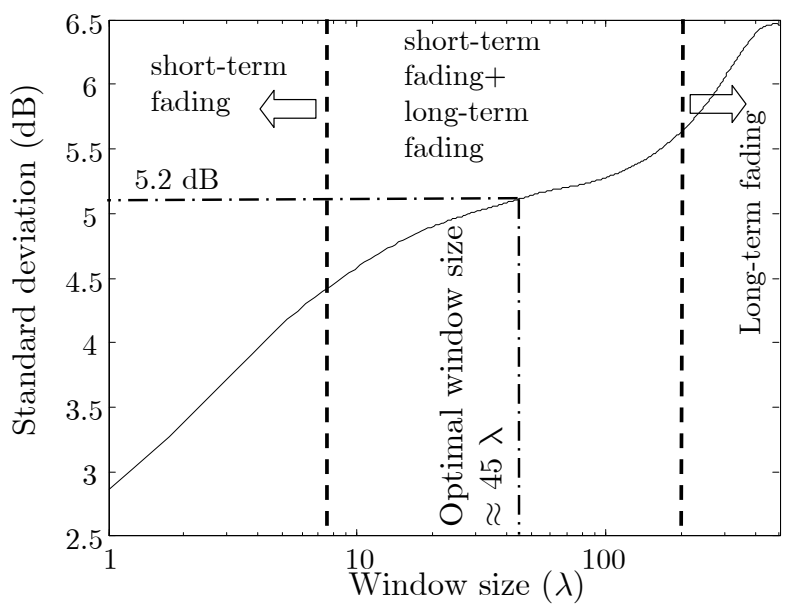

Fig. 6. Average standard deviation of the received signal in logarithmic units for a window size in $\lambda$ along the route \#5.

Fig. 6 shows the averaged standard deviation along the route \#5 as a function of the window size in $\lambda$. For window sizes less than $7 \lambda$, since the slope of the averaged standard deviation as a function of the logarithmic window size is approximately constant, we can assure that the local mean is almost constant. Thus, no appreciable shadowing effect is present in these window sizes. Furthermore the long-term variation prevails if the window size is higher than $200 \lambda$. Comparing the analytical standard deviation of (48) to the experimental result of Fig. 6, since the slope of the average standard deviation does not tend to 0 in any window sizes between the high slopes corresponding to small-scale and long-term fading (between 2 and 20, approximately). Hence 
the shadowing and the fast fading are substantially overlapped and the channel is non-stationary. In this paper we have estimated an average size window where the fast fading predominates. For the route $\# 5$ this optimal window size is approximately $45 \lambda$. The optimal windows size for each route

TABLE II

Optimal MEAN Window Size (MWS) FOR EXTRACTING Short-TERM Fading in Each Route as a Function of the WaVelength, $\lambda$.

\begin{tabular}{ccccccccc}
\hline Route & $\mathbf{\# 1}$ & $\mathbf{\# 2}$ & $\mathbf{\# 3}$ & $\mathbf{\# 4}$ & $\mathbf{\# 5}$ & $\mathbf{\# 6}$ & $\mathbf{\# 7}$ & $\mathbf{\# 8}$ \\
\hline MWS & $100 \lambda$ & $75 \lambda$ & $40 \lambda$ & $80 \lambda$ & $45 \lambda$ & $150 \lambda$ & $65 \lambda$ & $25 \lambda$ \\
\hline
\end{tabular}

using this method is shown in Table II. This mean window size varies substantially from $25 \lambda$ to $150 \lambda$ for routes \#8 and \#6, respectively. Fig. 7 shows the fading parameter, $m$, calculated

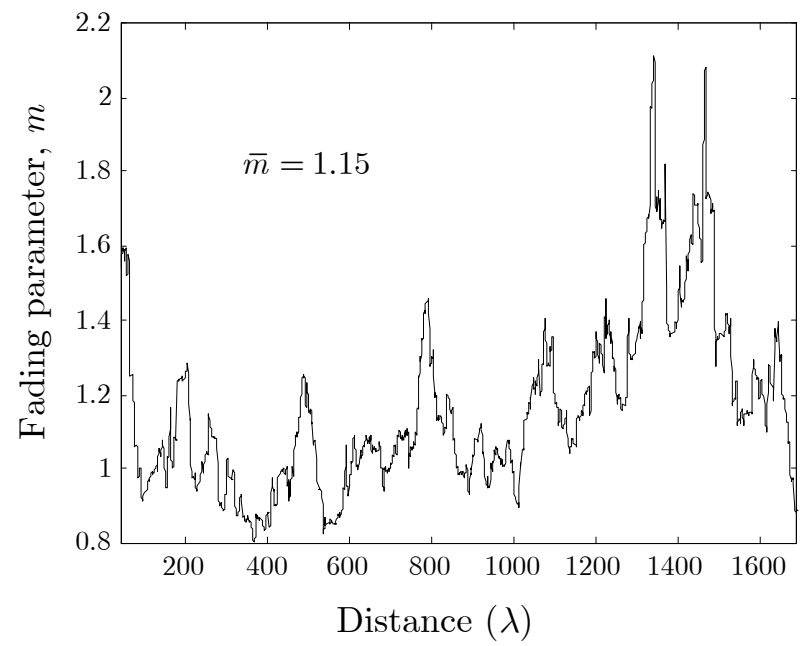

Fig. 7. Fading parameter, $m$, of the estimated Nakagami- $m$ distribution of the short-term fading along the route $\# 5$. The size of the window is $45 \lambda$. The mean of the fading parameter is $\bar{m}=1.15$.

over the average window size of $45 \lambda$ along the route $\# 5$ using the Lorenz estimator [22]. This fading parameter oscillates from 0.8 to 2.1. Note that fading parameters less than 1 correspond to situations as turning around a corner where the shadowing effect cannot be completely eliminated. The mean of the fading parameters calculated over this route is $\bar{m}=1.15$ which is very close to the estimated fading parameter for the Nakagami-lognormal distribution, $\hat{m}=1.14$, shown in Table III.

In Fig. 8, we have plotted the logarithmic CDF of the experimental distribution and the $\alpha-\mu$, Nakagami-lognormal, Rayleigh-lognormal, generalized- $K$ and $K$ distributions with the parameters estimated shown in Table III for the routes \#1, \#4 and \#8. The logarithmic CDFs have been obtained by substituting the inferred parameters of Table III in $F_{r}(\exp (\epsilon / A))$ given by (7), (21) and (35) for the Generalized- $K$, Nakagamilognormal and $\alpha-\mu$ distributions, respectively. Substituting $m=1$ in (7) and (21) and following the same procedure, we have derived the logarithmic CDFs for the $K$ and Suzuki distributions, respectively. For the route \#4 the differences between the CDF experimental and each CDF of the analyzed
TABLE III

Estimated PARAmeters of the Distributions For the ANALYZED ROUTES.

\begin{tabular}{|c|c|c|c|c|c|}
\hline & $\alpha-\mu$ & $\begin{array}{l}\text { Nakagami- } \\
\text { lognormal }\end{array}$ & Suzuki & Generalized- $K$ & $K$ \\
\hline $\begin{array}{l}\# 1 \\
4500 \\
\text { samples }\end{array}$ & $\begin{array}{l}\hat{\alpha}=0.45 \\
\hat{\mu}=5.01 \\
\hat{\Omega}=1.2 \cdot 10^{-3}\end{array}$ & $\begin{array}{l}\hat{m}=0.82 \\
\hat{\sigma}=6.33 \\
\hat{\eta}=-57.5\end{array}$ & $\begin{array}{l}\hat{\sigma}=7.13 \\
\hat{\eta}=-61.1\end{array}$ & $\begin{array}{l}\hat{m}=1.047 \\
\hat{M}=1.05 \\
\hat{\Omega}_{0}=3.8 \cdot 10^{-6}\end{array}$ & $\begin{array}{l}\hat{M}=0.73 \\
\hat{\Omega}_{0}=5.6 \cdot 10^{-6}\end{array}$ \\
\hline $\begin{array}{l}\# 2 \\
6266 \\
\text { samples }\end{array}$ & $\begin{array}{l}\hat{\alpha}=1.34 \\
\hat{\mu}=1.61 \\
\hat{\Omega}=7.1 \cdot 10^{-4}\end{array}$ & $\begin{array}{l}\hat{m}=1.03 \\
\hat{\sigma}=2.42 \\
\hat{\eta}=-62.7\end{array}$ & $\begin{array}{l}\hat{\sigma}=2.14 \\
\hat{\eta}=-65.6\end{array}$ & $\begin{array}{l}\hat{m}=1.038 \\
\hat{M}=3.51 \\
\hat{\Omega}_{0}=1.7 \cdot 10^{-7}\end{array}$ & $\begin{array}{l}\hat{M}=4.5 \\
\hat{\Omega}_{0}=1.3 \cdot 10^{-7}\end{array}$ \\
\hline $\begin{array}{l}\# 3 \\
3208 \\
\text { samples }\end{array}$ & $\begin{array}{l}\hat{\alpha}=1.17 \\
\hat{\mu}=1.98 \\
\hat{\Omega}=1.2 \cdot 10^{-3}\end{array}$ & $\begin{array}{l}\hat{m}=1.07 \\
\hat{\sigma}=2.78 \\
\hat{\eta}=-58.5\end{array}$ & $\begin{array}{l}\hat{\sigma}=2.17 \\
\hat{\eta}=-61.3\end{array}$ & $\begin{array}{l}\hat{m}=1.11 \\
\hat{M}=2.55 \\
\hat{\Omega}_{0}=7 \cdot 10^{-7}\end{array}$ & $\begin{array}{l}\hat{M}=4.4 \\
\hat{\Omega}_{0}=4.1 \cdot 10^{-7}\end{array}$ \\
\hline $\begin{array}{l}\# 4 \\
2419 \\
\text { samples }\end{array}$ & $\begin{array}{l}\hat{\alpha}=0.79 \\
\hat{\mu}=3.18 \\
\hat{\Omega}=4.9 \cdot 10^{-4}\end{array}$ & $\begin{array}{l}\hat{m}=1.06 \\
\hat{\sigma}=3.9 \\
\hat{\eta}=-65.6\end{array}$ & $\begin{array}{l}\hat{\sigma}=3.59 \\
\hat{\eta}=-68.5\end{array}$ & $\begin{array}{l}\hat{m}=1.35 \\
\hat{M}=1.37 \\
\hat{\Omega}_{0}=2.8 \cdot 10^{-7}\end{array}$ & $\begin{array}{l}\hat{M}=1.87 \\
\hat{\Omega}_{0}=2.1 \cdot 10^{-7}\end{array}$ \\
\hline $\begin{array}{l}\# 5 \\
3483 \\
\text { samples }\end{array}$ & $\begin{array}{l}\hat{\alpha}=0.58 \\
\hat{\mu}=5.3 \\
\hat{\Omega}=4 \cdot 10^{-4}\end{array}$ & $\begin{array}{l}\hat{m}=1.14 \\
\hat{\sigma}=4.51 \\
\hat{\eta}=-67.2\end{array}$ & $\begin{array}{l}\hat{\sigma}=3.87 \\
\hat{\eta}=-69.85\end{array}$ & $\begin{array}{l}\hat{m}=1.47 \\
\hat{M}=1.48 \\
\hat{\Omega}_{0}=2.1 \cdot 10^{-7}\end{array}$ & $\begin{array}{l}\hat{M}=1.67 \\
\hat{\Omega}_{0}=1.9 \cdot 10^{-7}\end{array}$ \\
\hline $\begin{array}{l}\# 6 \\
2832 \\
\text { samples }\end{array}$ & $\begin{array}{l}\hat{\alpha}=1.31 \\
\hat{\mu}=1.63 \\
\hat{\Omega}=1.5 \cdot 10^{-4}\end{array}$ & $\begin{array}{l}\hat{m}=1.02 \\
\hat{\sigma}=2.5 \\
\hat{\eta}=-76.1\end{array}$ & $\begin{array}{l}\hat{\sigma}=2.36 \\
\hat{\eta}=-79\end{array}$ & $\begin{array}{l}\hat{m}=1.026 \\
\hat{M}=3.27 \\
\hat{\Omega}_{0}=8.8 \cdot 10^{-9}\end{array}$ & $\begin{array}{l}\hat{M}=3.75 \\
\hat{\Omega}_{0}=7.6 \cdot 10^{-9}\end{array}$ \\
\hline $\begin{array}{l}\# 7 \\
2923 \\
\text { samples }\end{array}$ & $\begin{array}{l}\hat{\alpha}=1.68 \\
\hat{\mu}=1.22 \\
\hat{\Omega}=5.7 \cdot 10^{-4}\end{array}$ & $\begin{array}{l}\hat{m}=1.01 \\
\hat{\sigma}=1.63 \\
\hat{\eta}=-64.8\end{array}$ & $\begin{array}{l}\hat{\sigma}=1.43 \\
\hat{\eta}=-67.8\end{array}$ & $\begin{array}{l}\hat{m}=1.006 \\
\hat{M}=8.67 \\
\hat{\Omega}_{0}=4.1 \cdot 10^{-8}\end{array}$ & $\begin{array}{l}\hat{M}=10.03 \\
\hat{\Omega}_{0}=3.5 \cdot 10^{-8}\end{array}$ \\
\hline $\begin{array}{l}\# 8 \\
5361 \\
\text { samples }\end{array}$ & $\begin{array}{l}\hat{\alpha}=0.73 \\
\hat{\mu}=3.9 \\
\hat{\Omega}=1.3 \cdot 10^{-3}\end{array}$ & $\begin{array}{l}\hat{m}=1.15 \\
\hat{\sigma}=3.92 \\
\hat{\eta}=-57.3\end{array}$ & $\begin{array}{l}\hat{\sigma}=3.13 \\
\hat{\eta}=-59.9\end{array}$ & $\begin{array}{l}\hat{m}=1.48 \\
\hat{M}=1.49 \\
\hat{\Omega}_{0}=1.5 \cdot 10^{-6}\end{array}$ & $\begin{array}{l}\hat{M}=2.32 \\
\hat{\Omega}_{0}=1.2 \cdot 10^{-6}\end{array}$ \\
\hline
\end{tabular}

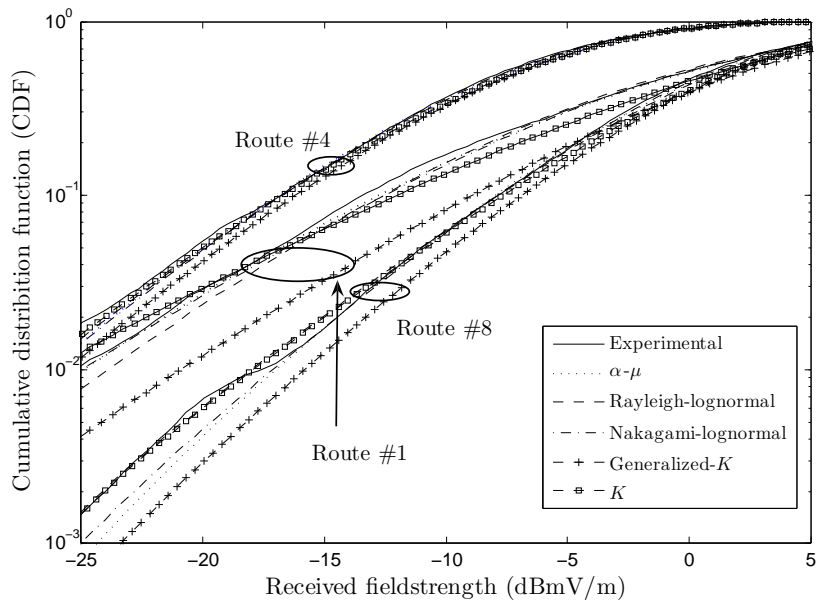

Fig. 8. Cumulative distribution functions of the experimental, $\alpha-\mu$ (generalized gamma), Nakagami-lognormal, Rayleigh-lognormal (Suzuki), Generalized- $K$ (gamma-gamma) and $K$ (Rayleigh-gamma) distributions using the log-moments for the routes \#1, \#4 and \#8.

distributions are low. The differences between experimental and some analyzed CDFs are significant in the routes \#1 and \#8. In particular the estimated generalized- $K$ CDF differs substantially from the experimental CDF. For the plotted CDFs, the $\alpha-\mu$ and Nakagami-lognormal CDFs fit the experimental CDF better than the other distributions although significant differences can be appreciated for the lower tails of the distribution for the route \#8. The K-S test of these distributions has been applied for all routes. Table IV summarizes the values of the confidence intervals of $5 \%$ and $1 \%, D_{5}$ and $D_{1}$, respectively. In bold letters, the values which provide less values of K-S statistic among the $\alpha-\mu$, Nakagami-lognormal, Rayleighlognormal, generalized- $K$ and $K$ distributions are shown. Note that the K-S test is calculated using the logarithmic CDFs. According to the K-S test results, the received field strength distribution which matches better the received field strength 
TABLE IV

KOLMOgOROV-SMIRnOV TEST FOR THE ANALYZED ROUTES.

\begin{tabular}{|c|c|c|c|c|c|}
\hline Distribution & $\alpha-\mu$ & N-L & Suzuki & G- $K$ & $K$ \\
\hline $\begin{array}{l}\text { Route \#1 } \\
D_{5}=0.0203 \\
D_{1}=0.0243\end{array}$ & 0.0181 & 0.0252 & 0.0261 & 0.132 & 0.0658 \\
\hline $\begin{array}{l}\text { Route \#2 } \\
D_{5}=0.0172 \\
D_{1}=0.0206\end{array}$ & 0.0091 & 0.0106 & 0.0110 & 0.0079 & 0.0116 \\
\hline $\begin{array}{l}\text { Route \#3 } \\
D_{5}=0.0240 \\
D_{1}=0.0288\end{array}$ & 0.0411 & 0.0273 & 0.0305 & 0.0503 & 0.0617 \\
\hline $\begin{array}{l}\text { Route \#4 } \\
D_{5}=0.0277 \\
D_{1}=0.0331\end{array}$ & 0.0185 & 0.0187 & 0.0209 & 0.0445 & 0.0391 \\
\hline $\begin{array}{l}\text { Route \#5 } \\
D_{5}=0.0230 \\
D_{1}=0.0276\end{array}$ & 0.0381 & 0.0279 & 0.0370 & 0.1128 & 0.0812 \\
\hline $\begin{array}{l}\text { Route \#6 } \\
D_{5}=0.0256 \\
D_{1}=0.0306\end{array}$ & 0.0175 & 0.0108 & 0.0152 & 0.0155 & 0.0193 \\
\hline $\begin{array}{l}\text { Route \#7 } \\
D_{5}=0.0252 \\
D_{1}=0.0301\end{array}$ & 0.0179 & 0.0124 & 0.0151 & 0.0154 & 0.0179 \\
\hline $\begin{array}{l}\text { Route \#8 } \\
D_{5}=0.0186 \\
D_{1}=0.0223\end{array}$ & 0.0267 & 0.0194 & 0.0346 & 0.0736 & 0.0641 \\
\hline
\end{tabular}

is in general the Nakagami-lognormal. This is the best-fitting distribution for the routes \#3, \#5-\#8. Nevertheless, the $\alpha-\mu$ distribution matches better the measured field strength in the routes \#1 and \#4, and the generalized- $K$ distribution is the best-fit distribution for the route \#2. Note that the K-S value for the generalized- $K$ distribution is considerable high for the routes \#4, \#5 and \#8 due to (12) and (13) are not fulfilled. Moreover the K-S condition is not accomplished in route \#5 for all analyzed distributions. In the routes \#3 and \#8, the K-S test is only fulfilled for a significance of $1 \%$.

\section{CONCLUSION}

In this paper, we have proposed a general method for estimating the parameters of the $\alpha-\mu$, Nakagami-lognormal, Suzuki, generalized- $K$ and $K$ distributions using the method of moments in logarithmic units. These distributions have been proposed to model the composite fast fading and shadowing effects. A novel and simple parameters estimator of the Nakagami-lognormal distribution has been derived. From a measurement campaign carried out in an urban environment of the city of Valencia, Spain, the parameters of these distributions have been estimated in eight routes with number of samples which oscillates from 2419 to 6266 . Using the K-S test, the Nakagami-lognormal is the best-fitting distribution in five routes. The second best distribution which matches the experimental measurements is the $\alpha-\mu$ being the best-fitting distribution in two routes. Moreover, to separate the fast fading and the shadowing we have proposed a procedure based on the Rathgeber method [37]. Using this procedure we can estimate the average window size in a route where the shadowing can be assumed neglected. The mean window sizes calculated in the routes vary considerably from $25 \lambda$ to $150 \lambda$.

\section{ACKNOWLEDGMENT}

The authors would like to thank the editor and reviewers their valuable comments which have enriched the quality of this paper. We would also express our gratitude to Dr. C. S. Withers, retired research statistician from the Applied Maths Group at Industrial Research Ltd, Lower Hutt, New Zealand, for his revision of the paper and his estimable remarks.

\section{REFERENCES}

[1] J. D. Parsons, Mobile Radio Propagation Channel, 2nd ed. Chichester, UK: John Wiley \& Sons, 2000.

[2] H. Suzuki, "A statistical model for urban radio propagation," IEEE Trans. Commun., vol. 25, pp. 673-680, Jul. 1977.

[3] S. R. Saunders and A. Aragón-Zavala, Antennas and Propagation for Wireless Communication Systems, 2nd ed. Chichester, UK: John Wiley \& Sons, 2007

[4] U. Charash, "Reception through Nakagami fading multipath channels with random delays," IEEE Trans. Commun., vol. 27, pp. 657-670, Apr. 1979.

[5] W. R. Braun and U. Dersch, "A physical mobile radio channel model," IEEE Trans. Veh. Technol., vol. 40, pp. 472-482, May 1991.

[6] T. Aulin, "Characteristics of a digital mobile radio channel," IEEE Trans. Veh. Technol., vol. 30, pp. 45-53, May 1981.

[7] J. Salo, L. Vuokko, H. M. El-Sallabi, and P. Vainikainen, "An additive model as a physical basis for shadow fading," IEEE Trans. Veh. Technol., vol. 56, pp. 13-26, Jan. 2007.

[8] G. L. Turin, F. D. Clapp, T. L. Johnston, S. B. Fine, and D. Lavry, "A statistical model of urban multipath propagation," IEEE Trans. Veh. Technol., vol. 21, pp. 1-9, Feb. 1972.

[9] O. Renaudin, V.-M. Kolmonen, P. Vainikainen, and C. Oestges, "Nonstationary narrowband MIMO inter-vehicle channel characterization in the 5 Ghz band," IEEE Trans. Veh. Technol., vol. 59, pp. 2007-2015, May 2010.

[10] G. Matz, "On non-WSSUS wireless fading channels," IEEE Trans. Wireless Commun., vol. 4, pp. 2465-2478, Sep. 2005.

[11] L. Bernadó, T. Zemen, J. Karedal, A. Paier, A. Thiel, O. Klemp, N. Czink, F. Tufvesson, A. Molisch., and C. Mecklenbräuker, "Multidimensional $K$-factor analysis for $\mathrm{V} 2 \mathrm{~V}$ radio channels in open sub-urban street crossings," in Proc. IEEE Personal, Indoor and Mobile Radio Communications, PIMRC, Sep. 2010, pp. 58-63.

[12] F. Hansen and F. I. Meno, "Mobile fading Rayleigh and lognormal superimposed," IEEE Trans. Veh. Technol., vol. 26, pp. 332-335, Nov. 1977.

[13] A. A. Abu-Dayya and N. C. Beaulieu, "Micro- and macrodiversity NCFSK (DPSK) on shadowed Nakagami-fading channels," IEEE Trans. Commun., vol. 42, pp. 2693-2702, Sep. 1994.

[14] T. T. Tjhung and C. C. Chai, "Bit error rate performance of $\pi / 4$ DQPSK for Nakagami-lognormal channels," Electron. Lett., vol. 34, pp. 625-627, Apr. 1998

[15] _ "Fade statistics in Nakagami-lognormal channels," IEEE Trans. Commun., vol. 47, pp. 1769-1772, Dec. 1999.

[16] G. Rafiq and M. Pätzold, "The influence of the severity of fading and shadowing on the statistical properties of the capacity of Nakagamilognormal channels," in Proc. IEEE Global Telecommunications Conference, 2008 (GLOBECOM'08), Nov. 2008.

[17] P. M. Shankar, "Outage probabilities in shadowed fading channels using a compound statistical model," IEE Proc. Commun., vol. 152, pp. 828832, Dec. 2005

[18] - "Error rates in generalized shadowed fading channels," Wireless Personal Communications, vol. 28, pp. 233-238, Feb. 2004.

[19] A. Abdi and M. Kaveh, "Comparison of DPSK and MSK bit error rates for $K$ and Rayleigh-lognormal fading distributions," IEEE Commun. Lett., vol. 4, pp. 122-124, Apr. 2000.

[20] _ " $K$ distribution: an appropriate substitute for Rayleigh-lognormal distribution in fading-shadowing wireless channels," Electron. Lett., vol. 34, pp. 851-852, Apr. 1998

[21] S. Nadarajaha and S. Kotz, "Compound statistical models for shadowed fading channels," Int. J. Electron. Commun. (AEÜ), vol. 62, pp. 138142, Feb. 2008.

[22] R. W. Lorenz, "Theoretical distribution functions of multipath propagation and their parameters for mobile radio communication in quasismooth terrain," in AGARD Terrain Profiles and Contours in Electromagnetic Wave Propagation, SAO/NASA ADS, Dec. 1979.

[23] C. S. Withers, "Moment estimates for the product of a log-normal and a power of a chi," Aust. \& N.Z. J. Stats., 1998.

[24] C. S. Withers and S. Nadarajah, "A generalized Suzuki distribution," Wireless Pers. Commun., vol. 62, pp. 807-830, Feb. 2012. 
[25] A. J. Coulson, A. G. Williamson, and R. G. Vaughan, "Improved fading distribution for mobile radio," IEE Proc. Commun., vol. 145, pp. 197202, Jun. 1998.

[26] V. Anastassopoulos, G. A. Lampropoulos, A. Drosopoulos, and M. Rey, "High resolution radar clutter statistics," IEEE Trans. Aerosp. Electron. Syst., vol. 35, pp. 43-60, Jan. 1999.

[27] N. J. Redding, "Estimating the parameters of the $K$ distribution in the intensity domain," Ph.D. dissertation, DSTO Electronics and Surveillance Research Laboratory, Australia, Jul. 1999. [Online]. Available: http://dspace.dsto.defence.gov.au/dspace/bitstream/1947/4203/1/DSTOTR-0839\%20PR.pdf

[28] M. D. Yacoub, "The $\alpha-\mu$ distribution: a general fading distribution," in Proc. IEEE Personal, Indoor and Mobile Radio Communications, PIMRC, Sep. 2002, pp. 629-633.

[29] — "The $\alpha-\mu$ distribution: a physical fading model for the Stacy distribution," IEEE Trans. Veh. Technol., vol. 56, pp. 122-124, Jan. 2007.

[30] E. W. Stacy, "A generalization of the gamma distribution," Ann. Math. Stat., vol. 33, pp. 1187-1192, Sep. 1962.

[31] G. L. Siqueira and E. J. A. Vásquez, "Local and global signal variability statistics in a mobile urban environment," Wireless Personal Communications, vol. 15 , pp. $61-78$, Oct. 2000.

[32] J. M. Nicolas and F. Tupin, "Gamma mixture modeled with second kind statistics: application to SAR image processing," in Proc. IEEE Geoscience and Remote Sensing Symposium, (IGARSS), vol. 4, Jun. 2002, pp. 2489-2491.

[33] C. Tison, J. M. Nicolas, and F. Tupin, "Accuracy of fisher distributions and log-moment estimation to describe amplitude distributions of high resolution SAR images over urban areas," in Proc. IEEE Geoscience and Remote Sensing Symposium, (IGARSS), vol. 3, Jun. 2003, pp. 19992001.

[34] G. K. Karagiannidis, N. C. Sagias, and T. Mathiopoulos, "The N*Nakagami fading channel model," in Proc. Int. Symp. Wireless Commun. Syst., Sep. 2005, pp. 185-189.

[35] J. B. Andersen, "Statistical distributions in mobile communications using multiple scattering," in Proc. General Assembly of the International Union of Radio Science, Aug. 2002.

[36] B. Bandemer, C. Oestges, N. Czink, and A. Paulraj, "Physically motivated fast-fading model for indoor peer-to-peer channels," Electron. Lett., vol. 45, pp. 515-517, May 2009.

[37] R. Rathgeber, "Comments on local mean estimation," in COST 231-TD18, Viena, Jan. 1992.

[38] M. Abramowitz, Handbook of Mathematical Functions, with Formulas, Graphs, and Mathematical Tables, 9th ed. New York, NY: Dover, 1972.

[39] P. M. Shankar, "A compound scattering pdf for the ultrasonic echo envelope and its relationship to $K$ and Nakagami distributions," IEEE Trans. Ultrason., Ferroelectr., Freq. Control, vol. 50, pp. 339-343, Mar. 2003.

[40] I. S. Gradshteyn and I. M. Ryzhik, Table of Integrals, Series and Products, 7th ed. San Diego, CA: Academic, 2007.

[41] P. S. Bithas, N. C. Sagias, P. T. Mathiopoulos, G. K. Karagiannidis, and A. A. Rontogiannis, "On the performance analysis of digital communications over generalized- $K$ fading channels," IEEE Commun. Lett., vol. 10, pp. 353-355, May 2006.

[42] G. Casella and R. L. Berger, Statistical Inference, 2nd ed. Pacific Grove, CA: Duxbury, Thomson, 2002.

[43] L. Rubio, J. Reig, and N. Cardona, "Evaluation of Nakagami fading behaviour based on measurements in urban scenarios," Int. J. Electron. Commun. (AË̈), vol. 61, pp. 135-138, Feb. 2007.

[44] E. W. Stacy and G. A. Mihram, "Parameter estimation for a generalized gamma distribution," Technometrics, vol. 7, pp. 349-358, Aug. 1965.

[45] J. Reig and L. Rubio, "On simple estimators of the $\alpha-\mu$ fading distribution," IEEE Trans. Commun., vol. 59, pp. 3254-3258, Dec. 2011.

[46] P. M. Shankar, "Statistical models for fading and shadowed fading channels in wireless systems: a pedagogical perspective," Wireless Personal Communications, vol. 60, pp. 191-213, Sep. 2011.

[47] A. Murase, I. C. Symington, and E. Green, "Handover criterion for macro and microcellular systems," in Proc. 41 st. IEEE Veh. Technol. Conf., May 1991, pp. 524-530.

[48] V. I. Tatarski, Wave Propagation in a Turbulent Medium. Traslated by Silverman. New York, NY: Mc-Graw-Hill, 1961.

[49] R. H. Clarke, "A statistical theory of mobile-radio reception," Bell System Technical Journal, pp. 957-1000, Jul-Aug 1968.

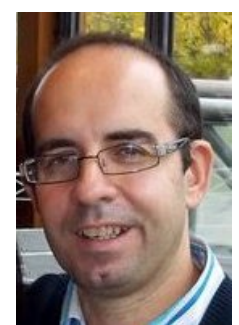

Juan Reig was born in Alcoy, Spain, in 1969. He received the M.S. and Ph.D. degrees in Telecommunications Engineering from the Technical University of Valencia, Spain, in 1993 and 2000, respectively. He has been a faculty member in the Department of Communications at the Technical University of Valencia, Spain since 1994, where he is now Associate Professor of Telecommunication Engineering. He is a member of the Grupo de Radiacin Electromagntica (GRE) of the Instituto de Telecomunicaciones y Aplicaciones Multimedia (iTEAM). His areas of interest include fading theory, diversity, ultra-wide band systems and vehicular communications in $\mathrm{V} 2 \mathrm{~V}$ and $\mathrm{V} 2 \mathrm{I}$ networks.

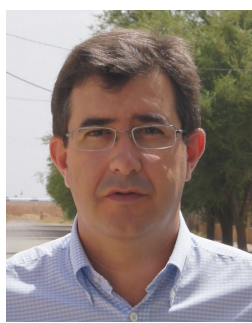

Lorenzo Rubio was born in El Ballestero, Albacete, Spain, in 1971. He received the Telecommunication Engineering and the Ph.D degrees from the Polytechnic University of Valencia (UPV), Spain, in 1996 and 2004 respectively. In 1996, he joined the Communications Department of the UPV, where he is now Associate Professor of wireless communications. He is a member of the Grupo de Radiacin Electromagntica (GRE) of the Instituto de Telecomunicaciones y Aplicaciones Multimedia (iTEAM). His main research interests are related to wireless communications. Specific current research topics include radiowave propagation, measurement and mobile time-varying channels modelling in vehicular applications, ultra-wideband (UWB) communication systems, MIMO systems and equalization techniques in digital wireless systems. Dr. Rubio was awarded by the Ericsson Mobile Communications Prize from the Spanish Telecommunications Engineer Association for his study on urban statistical radiochannels characterisation applied to wireless communications. 